\title{
DISPERSIÓN, SUPERVIVENCIA Y REPRODUCCIÓN DE LA PAVA ALIBLANCA Penelope albipennis TACZANOWSKI, 1877 (CRACIDAE) REINTRODUCIDA A SU HÁBITAT NATURAL EN PERÚ
}

\section{DISPERSAL, SURVIVAL AND REPRODUCTION OF REINTRODUCED WHITE-WINGED GUAN Penelope albipennis TACZANOWSKI, 1877 (CRACIDAE) TO ITS NATURAL HABITAT}

\author{
Fernando Angulo Pratolongo ${ }^{1}$
}

\begin{abstract}
Resumen
La pava aliblanca (Penelope albipennis) es un crácido endémico de los bosques secos del noroeste del Perú y se encuentra críticamente amenazado de extinción debido a la cacería y destrucción del hábitat. En el año 2000 se estableció el programa de reintroducción de esta especie. Este consistió en la liberación de individuos nacidos en cautiverio en el zoocriadero Barbara D’Achille en áreas donde la especie hubiera desaparecido con anterioridad y que en el presente aseguraran su conservación.

Entre Septiembre de 2001 y Julio de 2003 fueron liberados un total de 20 pavas aliblancas en el Área de Conservación Privada Chaparrí, Lambayeque, Perú. La adaptación de las aves al lugar fue medida mediante su dispersión, supervivencia y reproducción.

Se ha determinado en base a la distancia de dispersión, que es factible conectar la población reintroducida con las poblaciones silvestres aledañas. Con respecto a la supervivencia, evaluada a los dos años después de la liberación ha sido del 55\%. En cuanto a la reproducción, se ha obtenido tres polluelos nacidos en libertad de padres reintroducidos.

Palabras Claves: Pava Aliblanca, Penelope albipennis, reintroducción, dispersión, reproducción, supervivencia, crácidos, Chaparrí, bosque seco.
\end{abstract}

\begin{abstract}
The White-winged Guan (Penelope albipennis) is a cracid endemic to the Northwestern Peruvian dry forests that is critically endangered due to poaching pressure and habitat loss. In the year 2000, a species reintroduction program was established which consisted in the release of captive born and raised animals from the Barbara D'Achille captive breeding center. The guans were released in areas where the species had disappeared and that were safe for reintroduction and conservation.

Between September 2001 and July 2003, 20 White-winged Guans were released at the Chaparrí Private Conservation Area, Lambayeque, Perú. The bird's adaptation to the area was measured through its dispersion, survival and reproduction.

Based on the measured dispersion distance, it has been found that it is actually feasible to link the reintroduced population with the surrounding wild populations. Survival has been established at 55 $\%$, measured two years after the animals were released. With respect to reproduction, 3 wild-born chicks were born from reintroduced parents.
\end{abstract}

Key words: White-winged Guan, Penelope albipennis, reintroduction, dispersion, reproduction, survival, cracids, Chaparrí, dry forests.

\section{Introducción}

La reintroducción de individuos reproducidos en cautiverio al hábitat silvestre es una herramienta común usada para el rescate de especies en peligro de extinción. Este proceso consiste básicamente en tomar algunos animales silvestres y con ellos empezar un programa de crianza en cautiverio y luego, seleccionar los individuos más aptos para ser liberados en un área apta previamente escogida.

Este proceso se viene aplicando en la pava aliblanca Penelope albipennis Taczanowski, 1877, un crácido endémico de los Bosques Secos de la costa norte del Perú y críticamente amenazado de extinción (BirdLife International, 2000) debido a la destrucción de su hábitat por presión demográfica y a la cacería. La población silvestre en la actualidad se estima en menos de 300 individuos (Díaz \& del Solar, 1997), y el resultado del último censo, realizado en 1987, fue de 82 ejemplares (Ortíz \& Díaz, 1997).

El Proyecto de Conservación de la Pava Aliblanca, gerenciado por la Asociación Cracidae Perú, se creó con el objetivo principal de reproducir a la pava aliblanca en cautiverio y reintroducirla en lugares donde actualmente estuviera extinta, y así contribuir a su recuperación y evitar su extinción. El programa de reproducción en cautiverio funciona en el Zoocriadero Bárbara D’Achille, de propiedad del Sr. Gustavo del Solar, ubicado en el pueblo de Olmos, departamento 
de Lambayeque, en la costa norte de Perú. Allí se mantiene el plantel reproductor que provee los individuos para el Programa de Reintroducción. En este centro también se realizan investigaciones sobre biología reproductiva, sanidad y el comportamiento en cautiverio de la especie.

El programa de reintroducción de la pava aliblanca

Este programa, se basa en la guía para reintroducciones de la International Union for Conservation of Nature (IUCN, 1995) y se implementó desde el año 2000 en los territorios de la Comunidad Campesina "Santa Catalina de Chongoyape", parte de los cuales forman el Área de Conservación Privada Chaparrí de 34412 hectáreas (Angulo, 2002) (Figura $1)$.

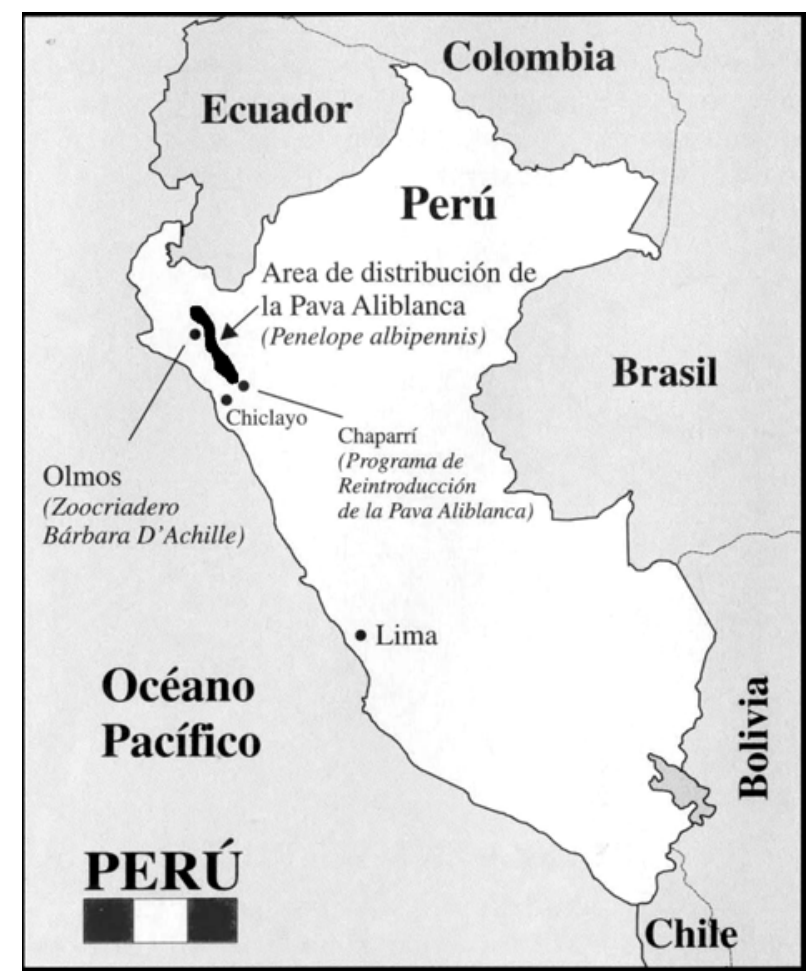

Figura 1. Área de distribución de la pava aliblanca (Penelope albipennis) y ubicación del programa de reproducción en cautiverio y reintroducción.

El principal objetivo del programa de reintroducción fue el establecer una población de pavas aliblancas viable en el largo plazo, que permita que los individuos reintroducidos se reproduzcan en libertad, de modo que la nueva población generada, que se puede considerar silvestre - se conecte con las poblaciones silvestres existentes en las zonas aledañas. Estas se encuentran distanciadas entre 12 y $19 \mathrm{~km}$ del área de reintroducción. Una de ellas, la población de la Zona Reservada de Laquipampa, se encuentra dentro de esta área protegida, creada especialmente para la conservación de la pava aliblanca. De esta forma, se pretende construir un corredor entre el área de reintroducción y los sectores antes señalados, que facilite el intercambio de material genético entre individuos, evitando una posible depresión genética de estas pequeñas poblaciones con tanto riesgo de desaparecer por el aislamiento geográfico (Angulo, 2003).

Una segunda finalidad del programa fue devolver a la comunidad de Santa Catalina un recurso, que bien manejado, resulta provechoso para los propios pobladores, usando a la pava aliblanca y a su hábitat como eje del flujo de turismo.

Los criterios usados para considerar el área como apta para este programa fueron los siguientes (IUCN, 1987; Balmford et al., 1996; Seddon et al., 1997):

1. La causa de que la especie desaparezca de ese lugar haya sido erradicada.

2. El lugar satisfaga los requerimientos de hábitat y se encuentre en suficiente extensión para la supervivencia de la especie.

El área seleccionada contó anteriormente con poblaciones silvestres de pava aliblanca, las cuales fueron exterminadas debido principalmente a la cacería. Al constituirse el área escogida como área protegida, se aseguró que la causa fuera erradicada, pues al ser de propiedad privada (comunal), son los mismos pobladores locales los comprometidos con la conservación de sus recursos y los que no permiten su cacería. Además, la misma causa de desaparición de la especie del área, ahora ya erradicada, asegura que el componente "hábitat" se encuentre en buen estado, pues no ha sido la destrucción de este la razón de la extirpación.

\section{Métodos \\ Reintroducción}

Las aves reintroducidas fueron seleccionadas del zoocriadero siguiendo los siguientes criterios:

- Individuos de las generaciones F1 o F2 (nacidos en cautiverio), que fuesen sexualmente maduros al momento de la liberación, con el objetivo de que durante la primera temporada de reproducción que tengan que experimentar en libertad, tuvieran disposición a la reproducción.

- Individuos con la mínima consanguinidad posible entre el grupo a liberar.

- Adecuada proporción de sexos para formar el grupo fundador. En este caso la proporción hembras : machos es 1:1 debido a la monogamia de la especie.

- Óptimo estado de salud (buen estado físico), para evitar el desarrollo y transmisión de enfermedades entre las mismas pavas y hacia otra fauna del lugar, y para garantizar un óptimo desenvolvimiento.

- El carácter de las aves. Estas no deben ser ni demasiado mansas ni demasiado ariscas.

Para facilitar la adaptación de los individuos a reintroducir al estado silvestre, se probaron tres diferentes métodos. 
El primero consistió en una fase de semicautiverio en la quebrada "Pavas" $\left(79^{\circ} 27\right.$ ' 33 " LO y $06^{\circ} 31$ ' 17 " LS), para lo que se construyó una jaula de malla de 70 x $30 \mathrm{~m}\left(2100 \mathrm{~m}^{2}\right)$ y 13 metros en su parte más alta. Esta jaula dejaba encerrado dentro de ella árboles y arbustos propios del hábitat y además, parte de un curso de agua permanente. Allí se mantuvieron 10 ejemplares por un tiempo variable (desde 01 hasta 15 meses) para que experimentaran el futuro hábitat, aprendieran a alimentarse, bebieran de fuentes naturales y ejercitaran sus miembros.

Como segundo método también de semicautiverio, se construyó frente al cerro Chaparrí $\left(79^{\circ} 28^{\prime} 23^{\prime \prime} \mathrm{LO}\right.$ y $06^{0} 32^{\prime} 07^{\prime}$ ' LS) una jaula de $25 \mathrm{~m}$ de largo, $5.5 \mathrm{~m}$ de ancho y una altura promedio de $2 \mathrm{~m}$, que ocupa una área útil de $138 \mathrm{~m}^{2}$. Ahí se mantuvieron 06 ejemplares por periodos entre 01 y 03 meses. La distancia entre ambas jaulas fue de $2.2 \mathrm{~km}$.

El tercer método consistió en la liberación de individuos provenientes del zoocriadero sin adaptación previa. Esto se llevó a cabo cuando hubo que sustituir individuos liberados mediante las estrategias anteriores.

Durante el semicautiverio se suministró alimento suplementario a las pavas a reintroducir (plátano, uva y tomate), especialmente durante la temporada de sequía. Se colocaron nidos artificiales (del mismo tamaño y forma que aquellos usados en el zoocriadero) en lugares seleccionados donde se creía podrían escoger las aves para anidar e incentivar la reproducción.

Además, las pavas fueron preparadas contra depredadores usando un gavilán acanelado Parabuteo unicinctus Temminck, 1824, entrenado, al cual se le hizo matar gallinas negras en presencia de las pavas. Este rapaz es un depredador natural tanto de individuos adultos como de polluelos de pava aliblanca en estado silvestre (Ortíz \& Díaz, 1997). Asimismo, se realizó un control anti-predadores colocando trampas donde se capturó y reubicó individuos de gato montés Oncifelis colocolo Molina, 1782, zorro costeño Pseudalopex sechurae Thomas, 1900 y muca o hurón Didelphis albiventris Lund, 1840.

En el aspecto sanitario, se realizaron pruebas para descartar las siguientes enfermedades, las cuales son las más comunes entre los galliformes en el área de influencia del centro de reproducción en cautiverio: Mycoplasma, Salmonella, Newcastle y Gumboro. También se realizaron exámenes para descartar la presencia de parásitos internos y externos. Las pruebas fueron hechas a las aves aun estando en el zoocriadero y fueron seleccionados y llevados al área de reintroducción aquellos individuos que dieron negativo a todas las pruebas.

Un total de 16 individuos fueron liberados entre el 23 de Septiembre y el 11 de Octubre de 2001, de los cuales, 09 fueron equipados con radiotransmisores
AVM tipo "Backpack" (mochila), de 23 gramos de peso y con vida útil de 1.5 años. Todos los individuos fueron marcados con una combinación de 2 anillos uno en cada pata-, de diferente color para individualizarlos.

Una vez liberadas las aves, se les monitoreó usando el método la telemetría y la observación directa con el objetivo de evaluar que metodología de adaptación de los individuos al medio es la que mejor funciona y poder medir el cumplimiento de los objetivos.

\section{Siguientes liberaciones}

Luego de la primera liberación del 2001 se continuó con el desarrollo del Programa de Reintroducción mediante la división transversal de la jaula de la quebrada "Pavas", donde en Enero de 2002, una hembra de una pareja reproductora del zoocriadero fue trasladada a la primera mitad junto con sus 2 polluelos de 02 días de nacidos.

En Marzo del mismo año, 06 juveniles fueron transportados a la segunda mitad de la jaula. Estos individuos tenían entre 10 y 13 meses de edad al momento del traslado. Tres de estas pavas murieron en el interior de la jaula de semicautiverio debido a la presencia de un zorro costeño. En Octubre de 2002, fue llevado desde el zoocriadero a la misma jaula, el macho (pareja de la hembra de la primera mitad y padre de los polluelos de 11 meses de edad en ese entonces), los cuales fueron separados de su madre y colocados junto a los tres juveniles en la segunda mitad.

Posteriormente, en Noviembre de 2002, dos juveniles (de 19 y 23 meses de edad a la fecha de liberación) fueron liberados en el sector "Pavas" y la pareja mantenida en la primera mitad de la jaula, padres de los polluelos separados, fue transportada al sector Chaparrí en Mayo de 2003 y luego liberada en este lugar, el macho en Junio y la hembra en Julio de 2003.

\section{Resultados}

Se ha hecho una evaluación preliminar de los resultados obtenidos en el Programa de Reintroducción hasta Octubre de 2003, basándose en tres criterios: Dispersión, Supervivencia y Reproducción.

En lo referente a la dispersión, se ha encontrado que en general, todos los individuos liberados interactúan fuertemente entre ellos para formar parejas, las cuales una vez logradas, permanecen en un territorio cuya extensión es variable en función a la disponibilidad de agua y alimento, especialmente en la época seca o de menor precipitación.

Se ha observado que los machos, al ser liberados, se ubican rápidamente en un territorio que reúna condiciones ecológicas óptimas, mientras que las hembras son más errantes y se mueven en áreas más extensas durante los primeros días posteriores a la liberación. Luego, se suelen conformar las parejas si es 
que existe afinidad entre los individuos. Cabe destacar que las pavas aliblancas formaron parejas tanto durante el semicautiverio como en libertad luego de la liberación.

La distancia que los individuos pueden moverse del lugar de liberación está determinada también por la disponibilidad de alimento y agua. En este caso, tres parejas (Parejas $\mathrm{N}^{\mathrm{o}} 1,2$ y 3 ) se asentaron en áreas con relativamente buena oferta de hábitat cerca de sus respectivos lugares de liberación, lo que impulsó al resto de individuos a moverse en diferentes direcciones en busca de hábitat apropiado.

Estos movimientos se dieron generalmente en solitario, pudiendo ser en un comienzo en pares. La distancia que las aves llegaron a alejarse de su lugar de la liberación varió desde los pocos metros hasta los 13 $\mathrm{km}$, habiéndose registrado dispersiones de 06, 07, 10, y 11 kilómetros, resultando esto en la posibilidad de que se de un refrescamiento genético.

Un hecho que se ha notado de esta experiencia es la capacidad de las pavas aliblancas de sobrevivir en áreas donde la formación vegetal predominante es el Algarrobal, que ocurre sobre terrenos generalmente planos y está dominada por el algarrobo (Prosopis spp.). Este tipo de hábitat es bastante diferente a los bosques heterogéneos de colinas que es el hábitat usado en la actualidad por las poblaciones silvestres, y anteriormente cubría extensas áreas en las partes bajas del actual rango de distribución de la pava aliblanca, pudiendo haber servido de corredor biológico para la dispersión de la especie en épocas remotas.

En lo referente a la supervivencia, se ha encontrado depredación de adultos en libertad por parte del gato montés y del hombre. El único caso de depredación por el hombre se dio fuera del área de conservación por un poblador que reportó no conocer la especie ni nunca haber escuchado hablar de ella.

En un caso se encontró un individuo muerto y devorado sin poder determinarse la causa del deceso. Se ha registrado también depredación de nidos por parte del zorro costeño y de la muca.

Del total de pavas liberadas en el primer grupo $(\mathrm{n}=$ 16), cinco tuvieron que ser retiradas al no tener una conducta apropiada para vivir en libertad. Del restante $(\mathrm{n}=11)$, dos tuvieron que ser retiradas debido a problemas sanitarios, lo que deja un total de nueve pavas. De este total, tres murieron, lo que el restante representa un $37.5 \%$ de permanencia sobre el total a los dos años de liberación, mientras que esta, en base a los individuos que permanecieron $(\mathrm{n}=11)$ en el área de liberación, es de $55 \%$ en el mismo lapso.

Con respecto a la reproducción, esta empezó a manifestarse ya desde el semicautiverio, cuando una pava construyó un nido dentro de la jaula de la quebrada "Pavas" e incubó tres huevos los cuales fueron infértiles. Los nidos artificiales fueron aceptados únicamente por la pareja $\mathrm{N}^{\circ} 1$, una de las tres que se establecieron inmediatamente después de la liberación. La hembra de esta pareja, asentada en el sector Chaparrí, utilizó un nido artificial colocado en un árbol, donde incubó su nidada la cual fue infértil, luego de que construyera su primer nido sobre el suelo y este fuera predado a los pocos días de ser depositados los huevos.

Las parejas $\mathrm{N}^{\mathrm{O}} 2$ y 3 , asentadas en el sector "Pavas", eligieron construir su propio nido. Hicieron varios intentos hasta que finalmente los establecieron de forma bastante similar al de una pava silvestre. El nido de la pareja $\mathrm{N}^{\circ} 2$, conteniendo tres huevos, fue predado por una muca y luego ningún otro intento de anidación fue mostrado por esta pareja.

La pareja $\mathrm{N}^{\mathrm{o}} 3$, construyó un nido en un árbol de cerezo cimarrón (Muntingia calabura) que medía $12 \mathrm{~m}$ de altura aproximadamente. El nido se encontraba a una altura de 05 metros sobre el nivel del suelo, cubierto de enredaderas, y construido de forma más elaborada que uno de pavas silvestres. Esta pareja tuvo éxito en este proceso, y a principios de Abril de 2002 nació un polluelo después de 30 días de incubación.

Este polluelo se convierte en el primer polluelo nacido en estado silvestre de pavas aliblancas reintroducidas y se le puede considerar técnicamente como silvestre. Este suceso es el comienzo del establecimiento de la nueva población de pavas aliblancas dentro del Área de Conservación Privada Chaparrí.

En Diciembre de 2002, la hembra de la pareja $N^{\circ} 3$, se echó nuevamente a incubar dos huevos en un nuevo nido construido por la pareja en un arbusto, a $1.5 \mathrm{~m}$ de altura. De estos dos huevos, solo uno fue fértil y el día 16 de enero nació un polluelo, el cual fue devorado tres días después presumiblemente por un sotillo Eira barbara Linnaeus, 1758 o un gato montés.

Dicha pareja intentó nuevamente reproducirse para lo cual construyó un nuevo nido y en los primeros días de Abril de 2003 nacieron 2 nuevos polluelos en libertad hijos de padres reintroducidos.

El éxito reproductivo de individuos reintroducidos, ya sean estos reproducidos en cautiverio o no, es un indicador del éxito de la implementación de un programa de reintroducción (Sanz \& Grajal, 1988).

Esto demuestra asimismo que las pavas aliblancas reintroducidas construyen un nuevo nido para cada evento reproductivo, pues la pareja $\mathrm{N}^{\circ} 3$ incubó sus tres nidadas en tres nidos diferentes.

Tratando exclusivamente sobre la dispersión (en este caso la separación del grupo familiar) de los juveniles nacidos en libertad hijos de padres reintroducidos, se ha encontrado que el primer polluelo (nacido en 2002), se separó de sus padres a los 11 meses de edad y se unió a dos pavas jóvenes liberadas anteriormente, formando un pequeño grupo que se dedicó a explorar sus alrededores, dispersándose hasta $04 \mathrm{~km}$ del lugar de nacimiento. En el caso de los dos polluelos de la segunda camada (nacidos en 2003), estos se separaron de los padres a los seis meses de 
edad, uniéndose igualmente, al grupo de juveniles que integraba su hermano mayor.

Este comportamiento pareciera ser normal, pues los jóvenes deben buscar pareja y nuevos territorios para cuando estén maduros sexualmente. En el primer caso (11 meses), la separación coincide justamente con la llegada de la nueva temporada reproductiva de los padres, mientras que en el segundo caso (06 meses), esta ocurrió antes de la llegada de la siguiente temporada reproductiva, por lo que se presume que no ocurrió por presión de los padres.

Tentativamente, esto se puede explicar con la teoría de que el primer polluelo, por ser uno solo, se encontraba mas apegado a los padres y se separó de estos debido a la presión de los progenitores, mientras que los siguientes dos polluelos, por el hecho de estar acompañados entre ellos mismos, y además, debido a que en los alrededores ya existía un grupo de pavas juveniles, facilitó su separación de los padres pues contaron con un grupo al cual integrarse.

Esta explicación de los mecanismos de separación del grupo familiar que pretende descifrar la forma en que esta especie mantiene o amplia su rango de extensión y los factores que la afectan, con el objetivo de poder manejar este factor para el establecimiento de corredores biológicos, es solo tentativa y teórica.

En cuanto a sanidad, se encontró que una pava sufrió de una lesión en una pata lo que le impedía desplazarse correctamente, mientras que otra fue atacada por una infección al oído (otitis), por lo que ambas tuvieron que ser retiradas del programa y sustituidas por nuevos ejemplares.

\section{Conclusiones y recomendaciones}

1. Sería factible el conectar la población reintroducida con las poblaciones silvestres aledañas (dispersión máxima $=13 \mathrm{~km}$ vs. población aledaña a menor distancia $=12 \mathrm{~km}$ ).

2. Los juveniles forman grupos que se mueven constantemente, habiendo llegado a alejarse hasta 04 $\mathrm{km}$ de su lugar de nacimiento o liberación.

3. El mecanismo de separación del grupo familiar padres-hijos se da al menos entre los 06 y 11 meses de edad.

4. La formación vegetal Algarrobal puede mantener poblaciones de pavas aliblancas al menos en bajas densidades y por periodos cortos de tiempo. Pero el Algarrobal es actualmente intensamente usado por los pobladores locales para pastoreo de ganado, extracción de leña y carbón y conversión en áreas agrícolas, por lo que probablemente en la actualidad no sea hábitat apropiado para las pavas debido a la presencia humana.

5. No se ha encontrado mayores diferencias en lo referente a la supervivencia basándose en el tiempo de permanencia en semicautiverio o la ausencia de este. Es recomendable tener a los individuos el menor tiempo en semicautiverio pues hay riesgos de predación en esta condición.

6. Durante la fase de semicautiverio se recomienda mantener algunos individuos de manera permanente para servir de "maestros" para los individuos recién arribados pues enseñan a estos de donde beber, que comer, donde dormir, etc.

7. La supervivencia en base a los individuos que permanecieron $(\mathrm{n}=11)$ en el área de liberación es de $55 \%$ al cabo de dos años de libertad.

8. La reproducción de individuos reintroducidos es factible. Estas aves pueden comportarse en ese aspecto como silvestres, al construir nidos y proteger sus polluelos como tales.

9. El hecho de haber obtenido un primer polluelo a los 06 meses de la liberación de los padres y al año siguiente dos polluelos más, es un hecho alentador y que da una esperanza de poder salvar esta especie de la extinción.

10. La labor de difusión y educación de la población en el área donde se realizará una reintroducción y zonas aledañas debe ser intensa antes y durante la fase de implementación.

11. Se recomienda el monitoreo de los individuos liberados dentro de un programa de reintroducción, pues permite evaluar la validez de los métodos usados y rediseñar el programa en el futuro.

\section{Agradecimientos}

A Don Gustavo del Solar por la confianza depositada. Al Sr. Heinz Plenge, al personal de la Asociación Cracidae Perú, del Zoocriadero Bárbara D'Achille y del Programa de Reintroducción. A la Comunidad Santa Catalina de Chongoyape. A la Fundación Backus por el valioso apoyo económico. Al Lincoln Park Zoo Neotropic Fund por apoyar económicamente en parte de este proyecto. A Idea Wild por colaborar con parte del equipo de telemetría. A Fernando González-García y Fabiola Riva por la paciente revisión de los primeros borradores. A todos ellos, mi más profundo agradecimiento por ayudar a hacer este proyecto una realidad.

\section{Literatura citada}

Angulo F. 2002. Área de Conservación Privada Chaparrí. Plan Maestro.

2003. Re-introduction of the White-winged Guan in north-west Peru. Reintroduction NEWS. Newsletter of the IUCN/SSC Re-introduction Specialist Group, Abu Dhabi, UAE. 23: 19-20.

Balmford A., Mace G.M. \& Leader-Williams N. 1996. Designing the Ark: Setting Priorities for Captive Breeding. Conservation Biology. 10 (3): 719-727.

BirdLife International. 2000. Threatened birds of the World. Barcelona, Spain \& Cambridge, U.K.: Lynx Editions and BirdLife International.

Díaz V. \& del Solar G. 1997. Resultados Parciales del Plan Integral para Salvar a la Pava Aliblanca 
(Penelope albipennis). Pp. 467-471 En: The Cracidae: their Biology and Conservation (S.D. Strahl, S. Beaujon, D.M. Brooks, A.J. Begazo, G. Sedaghatkish and F. Olmos). Hancock House Publ.

IUCN. 1987. The IUCN position statement on translocation of living organism: Introduction, reintroductions and re-stocking. IUCN Gland, Switzerland.

1995. IUCN Guidelines for Reintroductions. IUCN Gland, Switzerland.

Ortíz E. \& Díaz V. 1997. Estudio de Campo y Reevaluación de la Población de Pava Aliblanca
(Penelope albipennis). Pp. 218-232 In: The Cracidae: their Biology and Conservation (S. D. Strahl, S. Beaujon, D.M. Brooks, A.J. Begazo, G. Sedaghatkish and F. Olmos). Hancock House Publ. Sanz V. \& Grajal A. 1998. Successful Reintroduction of Captive-Raised Yellow-Shouldered Amazon Parrots on Margarita Island, Venezuela. Conservation Biology. 12 (2): 430-441.

Seddon P.J. \& Soorae P.S. 1999. Guidelines for Subspecific Substitutions in Wildlife Restoration Projects. Conservation Biology. 13 (1): 177-184.

\footnotetext{
${ }^{1}$ Director del Zoocriadero Bárbara D’Achille \& Programa de Reintroducción de la Pava Aliblanca. Asociación Cracidae Perú.

Torres Paz 708

Chiclayo, Lambayeque, Perú

Correo electrónico: chamaepetes@gmail.com
} 